\title{
Evaluation of energy balance closure correction methods for multiple eddy-covariance sites in different biomes
}

\author{
Matthias Mauder ${ }^{1}$ and the Energy-balance closure correction evaluation team ${ }^{*}$ \\ ${ }^{1}$ Karlsruhe Institute of Technology KIT/IMK-IFU, Institute for Meteorology and Climatology - Atmospheric Environmental \\ Research, Garmisch-Partenkirchen, Germany (matthias.mauder@kit.edu) \\ ${ }^{*}$ A full list of authors appears at the end of the abstract
}

The apparent lack of surface energy balance closure is one of the most crucial challenges in the measurement of biosphere-atmosphere exchange. In principle, this issue can have a variety of potential reasons, including instrumental errors and errors introduced in the data processing chain. In addition, secondary circulations have been identified as one of the main reasons for a non-closure of the surface energy balance, since the related energy transport cannot be captured by common eddy-covariance tower flux measurements. When present, neglecting this process will result in an underestimation of turbulent fluxes. Secondary circulations can, however, be represented by means of large-eddy simulations, which have been employed to develop a novel semi-empirical model to correct for the missing large-scale flux (De Roo et al. 2018, DOI 10.1371/journal.pone.0209022). In this study, we compare the results of this process-based method with two other previously published bulk-correction methods (Mauder et al. 2013, DOI 10.1016/j.agrformet.2012.09.006; Charuchittipan et al. 2014, DOI 10.1007/s10546-014-9922-6). These three correction methods are applied for multiple sites in different biomes around the world. Independent data of energy fluxes from these sites are used to assess which of these methods leads to the most reliable results, and we discuss the limitations of these corrections methods with respect to meteorological conditions and site characteristics, such as measurement height, the landscape-scale heterogeneity and terrain complexity.

Energy-balance closure correction evaluation team: Matthias Mauder, David Johnson, Gerardo Fratini, Daniel Berveiller, Aurore Brut, Pramit Kumar Deb Burman, Arnaud Carrara, Supriyo Chakraborty, Kuan-Yin Chen, Simon Drollinger, Milan Fischer, Anne Griebel, Atul K. Jain, Georg Jocher, Natascha Kljun, Anne Klosterhalfen, Natalia Kowalska, Hjalmar Laudon, Luca Belelli Marchesini, Ivan Mammarella, Daniel Metzen, Leonardo Montagnani, Sandhya K. Nair, Mats Nilsson, Asko Normets, Matej Orsag, Marian Pavelka, Matthias Peichl, Elise Pendall, Prajaya Prajapati, Debora Regina Roberti, Humberto Rocha, Eyal Rotenberg, Marko Stojanović, Paul Stoy, Efrat Schwartz, Will Woodgate, Dan Yakir 